Network Working Group

Request for Comments: 5067

Category: Informational
S. Lind

AT\&T Labs

P. Pfautz

$\mathrm{AT} \& \mathrm{~T}$

November 2007

\title{
Infrastructure ENUM Requirements
}

Status of This Memo

This memo provides information for the Internet community. It does not specify an Internet standard of any kind. Distribution of this memo is unlimited.

Abstract

This document provides requirements for "infrastructure" or "carrier" ENUM (E.164 Number Mapping), defined as the use of RFC 3761 technology to facilitate interconnection of networks for E.164 number addressed services, in particular but not restricted to VoIP (Voice over IP.)

Table of Contents

1. Infrastructure ENUM . . . . . . . . . . . . . . . . . . . . 1 1.1. Definition . . . . . . . . . . . . . . . . . . . . . . . 1

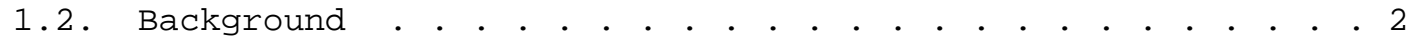

2. Terminology . . . . . . . . . . . . . . . . . . . . . . 3

3. Requirements for Infrastructure ENUM . . . . . . . . . . . . 3

4. Security Considerations . . . . . . . . . . . . . . . . . . . 4

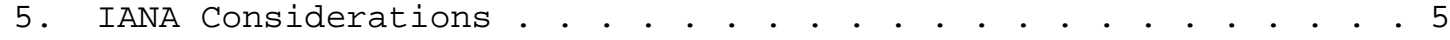

6. Normative References . . . . . . . . . . . . . . . . 5

1. Infrastructure ENUM

1.1. Definition

Infrastructure ENUM is defined as the use of the technology in RFC 3761 [1] by the carrier-of-record (as defined below) for a specific E.164 number [2] to publish the mapping of the E.164 number into a URI [3] that identifies a specific point of interconnection to that service provider's network. It is separate from any URIs that the end user, who registers their E.164 number, may wish to associate with that E.164 number. 
"Infrastructure ENUM" is distinguished from "End User ENUM", defined in RFC3761 [1], in which the entity or person having the right to use a number has the sole discretion about the content of the associated domain and thus the zone content. From a domain registration perspective, the end user number assignee is thus the registrant. Within the infrastructure ENUM namespace, we use the term "carrierof-record" for the entity having discretion over the domain and zone content and acting as the registrant. The "carrier-of-record" is:

- The Service Provider to which the E.164 number was allocated for end user assignment, whether by the National Regulatory Authority (NRA) or the International Telecommunication Union (ITU), for instance, a code under "International Networks" (+882) or "Universal Personal Telecommunications (UPT)" (+878) or,

o if the number is ported, the service provider to which the number was ported, or

o where numbers are assigned directly to end users, the service provider that the end user number assignee has chosen to provide a Public Switched Telephone Network/Public Land Mobile Network (PSTN/ PLMN) point-of-interconnect for the number.

It is understood that the definition of carrier-of-record within a given jurisdiction is subject to modification by national authorities.

\subsection{Background}

Voice service providers use E.164 numbers currently as their main naming and routing vehicle. Infrastructure ENUM in e164.arpa or another publicly available tree allows service providers to link Internet-based resources such as URIs to E.164 numbers. This allows service providers, in addition to interconnecting via the PSTN/PLMN (or exclusively), to peer via IP-based protocols. Service providers may announce all E.164 numbers or number ranges they host, regardless of whether the final end user device is on the Internet, on IP-based open or closed Next Generation Networks (NGNs), or on the PSTN or PLMN, provided that an access point of some type to the destination service provider's network is available on the Internet. There is also no guarantee that the originating service provider querying infrastructure ENUM is able to access the ingress network element of the destination provider's network. Additional peering and accounting agreements requiring authentication may be necessary. The access provided may also be to a shared network of a group of providers, resolving the final destination network within the shared network. 


\section{Terminology}

The key words "MUST", "MUST NOT", "REQUIRED", "SHALL", "SHALL NOT", "SHOULD", "SHOULD NOT", "RECOMMENDED", "MAY", and "OPTIONAL" in this document are to be interpreted as described in BCP 14, RFC2119 [4].

3. Requirements for Infrastructure ENUM

1. Infrastructure ENUM SHALL provide a means for a provider to populate DNS resource records (RRs) for the E.164 numbering resources for which it is the carrier-of-record in a single common publicly accessible namespace. The single common namespace ultimately designated may or may not be the same as that designated for End User ENUM (e164.arpa.) The FullyQualified Domain Name (FQDN) in the resulting resource records will not necessarily belong to or identify the carrier-of-record.

2. Queries of infrastructure ENUM fully qualified domain names MUST return a result, even if the result is Refused ( $\mathrm{RCODE}=5$ ). Queries must not be rejected without response, e.g., based on access control lists.

3. Infrastructure ENUM SHALL support RRs providing a URI that can identify a point of interconnection for delivery to the carrierof-record of communications addressed to the E.164 number.

4. Infrastructure ENUM SHOULD be able to support an Internet Registry Information Service (IRIS) [5] capability that allows qualified parties to obtain information regarding the E.164 numbering resources and the corresponding carrier-of-record. Determination of what information, if any, shall be available which parties for geographic numbers is a national matter.

5. Implementation of infrastructure ENUM MUST NOT restrict the ability of an end user, in a competitive environment, to choose a Registrar and/or name server provider for End User ENUM registrations.

6. The domain name chosen for infrastructure ENUM and any parent domains MUST be hosted on name servers that have performance characteristics and supporting infrastructure that is comparable to those deployed for the Internet root name servers. Those name servers for infrastructure ENUM should be configured and operated according to the guidelines described in [6].

7. Infrastructure ENUM MUST meet all reasonable privacy concerns about visibility of information over which an end user has no control. It should, for example, support mechanisms to prevent 
discovery of unlisted numbers by comparison of ENUM registrations against directory listings, or inadvertent disclosure of user identity.

8. Proposed implementations of infrastructure ENUM SHOULD:

A. Minimize changes required to existing requirements that are part of RFC 3761 .

B. Work with open as well as closed numbering plans.

C. Not require additional functionality of resolvers at large though they may require additional functionality in service provider resolvers that would make use of infrastructure ENUM.

D. Minimize the number of lookups required to obtain as many NAPTR (Naming Authority Pointer) records (end user and infrastructure) as possible.

E. Minimize knowledge of the numbering plan required of resolvers making use of Infrastructure ENUM.

F. Maximize synergies with End User ENUM.

G. Support interworking with private ENUM trees. (In this context, a private ENUM tree is one that is not under e164.arpa or whatever namespace is chosen for infrastructure ENUM but uses instead a privately held domain.)

4. Security Considerations

Existing security considerations for ENUM (detailed in [1]) still apply. Since infrastructure ENUM involves carriers where RFC 3761 mainly considered indviduals, implementations meeting these requirements SHOULD reconsider the RFC 3761 security model given this difference in actors concerned. Note that some registration validation issues concerning End User ENUM may not apply to infrastructure ENUM. Where the Tier 1 registry is able to identify the provider serving a number, e.g., based on industry data for number block assignments and number portability, registration might be more easily automated and a separate registrar not required.

Some parties have expressed concern that an infrastructure ENUM could compromise end user privacy by making it possible for others to identify unlisted or unpublished numbers based on their registration in ENUM. This can be avoided if providers register all of the their allocated (as opposed to assigned) numbers. Unassigned numbers 
should be provisioned to route to the provider's network in the same fashion as assigned numbers and only then provide an indication that they are unassigned. In that way, provider registration of a number in ENUM provides no more information about the status of a number than could be obtained by dialing it.

Implementers should take care to avoid inadvertent disclosure of user identities, for example, in the URIs returned in response to infrastructure ENUM queries.

5. IANA Considerations

This document includes no actions to be taken by IANA. The architecture ultimately chosen to meet the requirements may require IANA actions.

6. Normative References

[1] Faltstrom, P. and M. Mealling, "The E.164 to Uniform Resource Identifiers (URI) Dynamic Delegation Discovery System (DDDS) Application (ENUM)", RFC 3761, April 2004.

[2] International Telecommunication Union-Telecommunication Standardization Sector, "The International Public Telecommunication Numbering Plan", Recommendation E.164", February 2005 .

[3] Berners-Lee, T., Fielding, R., and L. Masinter, "Uniform Resource Identifier (URI): Generic Syntax", STD 66, RFC 3986, January 2005 .

[4] Bradner, S., "Key words for use in RFCs to Indicate Requirement Levels", BCP 14, RFC 2119, March 1997.

[5] Newton, A. and M. Sanz, "IRIS: The Internet Registry Information Service (IRIS) Core Protocol", RFC 3981, January 2005.

[6] Bush, R., Karrenberg, D., Kosters, M., and R. Plzak, "Root Name Server Operational Requirements", BCP 40, RFC 2870, June 2000. 


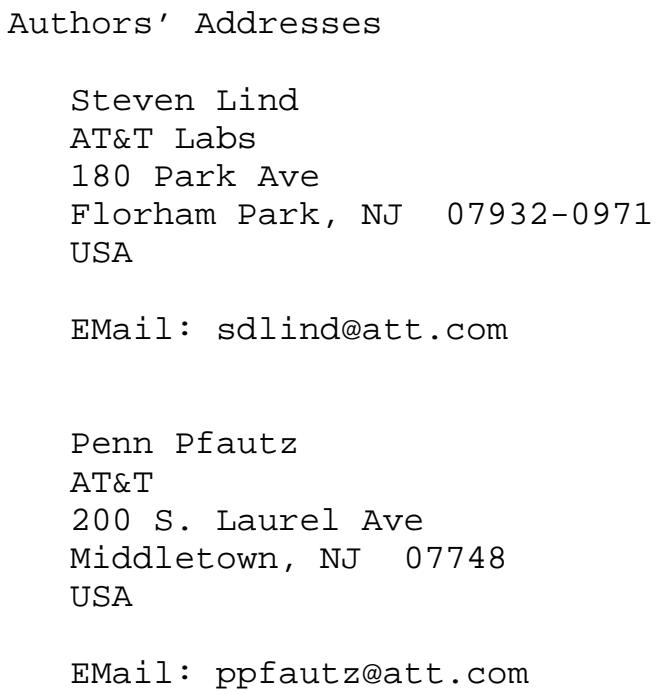


Full Copyright statement

Copyright (C) The IETF Trust (2007).

This document is subject to the rights, licenses and restrictions contained in BCP 78, and except as set forth therein, the authors retain all their rights.

This document and the information contained herein are provided on an "AS IS" basis and THE CONTRIBUTOR, THE ORGANIZATION HE/SHE REPRESENTS OR IS SPONSORED BY (IF ANY), THE INTERNET SOCIETY, THE IETF TRUST AND THE INTERNET ENGINEERING TASK FORCE DISCLAIM ALL WARRANTIES, EXPRESS OR IMPLIED, INCLUDING BUT NOT LIMITED TO ANY WARRANTY THAT THE USE OF THE INFORMATION HEREIN WILL NOT INFRINGE ANY RIGHTS OR ANY IMPLIED WARRANTIES OF MERCHANTABILITY OR FITNESS FOR A PARTICULAR PURPOSE.

Intellectual Property

The IETF takes no position regarding the validity or scope of any Intellectual Property Rights or other rights that might be claimed to pertain to the implementation or use of the technology described in this document or the extent to which any license under such rights might or might not be available; nor does it represent that it has made any independent effort to identify any such rights. Information on the procedures with respect to rights in RFC documents can be found in BCP 78 and BCP 79 .

Copies of IPR disclosures made to the IETF Secretariat and any assurances of licenses to be made available, or the result of an attempt made to obtain a general license or permission for the use of such proprietary rights by implementers or users of this specification can be obtained from the IETF on-line IPR repository at http://www.ietf.org/ipr.

The IETF invites any interested party to bring to its attention any copyrights, patents or patent applications, or other proprietary rights that may cover technology that may be required to implement this standard. Please address the information to the IETF at ietf-ipreietf.org. 